\title{
On the Benefits of Obtaining Surface Topography and Volume Structure Information by Correlative S(T)EM in a Scanning Electron Microscope
}

\author{
Cheng Sun, Erich Müller and Dagmar Gerthsen
}

Laboratory for Electron Microscopy (LEM), Karlsruhe Institute of Technology (KIT), 76131

Karlsruhe, Germany.

Scanning transmission electron microscopy (STEM) with low-energy electrons is well suited to analyze beam-sensitive materials and to obtain enhanced material contrast of weakly scattering objects. Low-energy STEM is predominantly performed by operating transmission electron microscopes at low acceleration voltages. Alternatively, scanning electron microscopes with electron energies $\mathrm{E}_{0} \leq 30 \mathrm{keV}$ can be fitted with a STEM detector and provide the advantage that surface topography imaging by scanning electron microscopy (SEM) is inherently available. This facilitates correlative (simultaneous) acquisition of information on surface topography and volume structure from the same region of electron transparent specimens. Only few studies, all performed at 200 $\mathrm{keV}$, have focused on the benefits of combined SEM surface topography and bulk STEM imaging, e.g., [1].

A Thermo Fisher DualBeam Helios G4 FX with improved resolution was used in this work. It is equipped with a STEM detector, a double-tilt holder for TEM specimens and a CCD-camera for acquiring on-axis transmission electron diffraction patterns. For optimization of imaging conditions, the primary electron energy and the detection angle range can be easily changed without elaborate realignment of the microscope.

Figure 1 shows $\mathrm{Pt}$ nanoparticles (NPs) on a porous $\mathrm{Al}_{2} \mathrm{O}_{3}$ carrier for catalysis. The secondaryelectron (SE) SEM image Figure 1a shows topography while material contrast by backscatteredelectron (BSE) SEM imaging (Figure 1b) reveals larger Pt particles at the top and the lower surface of the agglomerate. The same region is imaged by high-angle annular dark-field (HAADF-)STEM (Figure 1c) where porosity of $\mathrm{Al}_{2} \mathrm{O}_{3}$ is visualized. The larger Pt particles show dark contrast due to pronounced large-angle scattering beyond the detection angle range of the HAADF STEM detector. High-magnification HAADF-STEM imaging (Figure 1d) from a thin region (frame in Figures 1a,c), shows the presence of Pt NPs with a size clearly below $3 \mathrm{~nm}$ while topography contrast of this region is seen in the insert of Figure 1a.

Figure 2 shows SEM and low-energy STEM images of a thin section of an A549 cell incubated with $\mathrm{SiO}_{2}$ particles. Membranes, cell organelles and $\mathrm{SiO}_{2} \mathrm{NPs}$ contained in a vesicle show massthickness contrast in bright-field (BF-) and annular dark-field (ADF-)STEM images taken simultaneously within a single scan (Figures 2a,b). High-magnification ADF-STEM reveals the porous structure of the $\mathrm{SiO}_{2} \mathrm{NP}$ (Figure 2c) consistent with the SE-SEM image of the surface of another $\mathrm{SiO}_{2}$ particle in Figure 2d.

Correlative SEM and STEM in a scanning electron microscope is a powerful and flexible method for comprehensive analysis of a large variety of samples with extended capabilities in the future. [2, 3]

\section{References:}

[1] H Inada et al, Ultramicroscopy 111 (2011), p. 865.

[2] We thank Dr Maria Casapu, Andreas Gremminger, Prof Dirk Grunwaldt (Institute for Chemical Technology and Polymer Chemistry, Karlsruhe Institute for Technology (KIT)) for providing the $\mathrm{Pt} / \mathrm{Al}_{2} \mathrm{O}_{3}$ sample. 
[3] The authors acknowledge funding from the DFG (Deutsche Forschungsgemeinschaft).
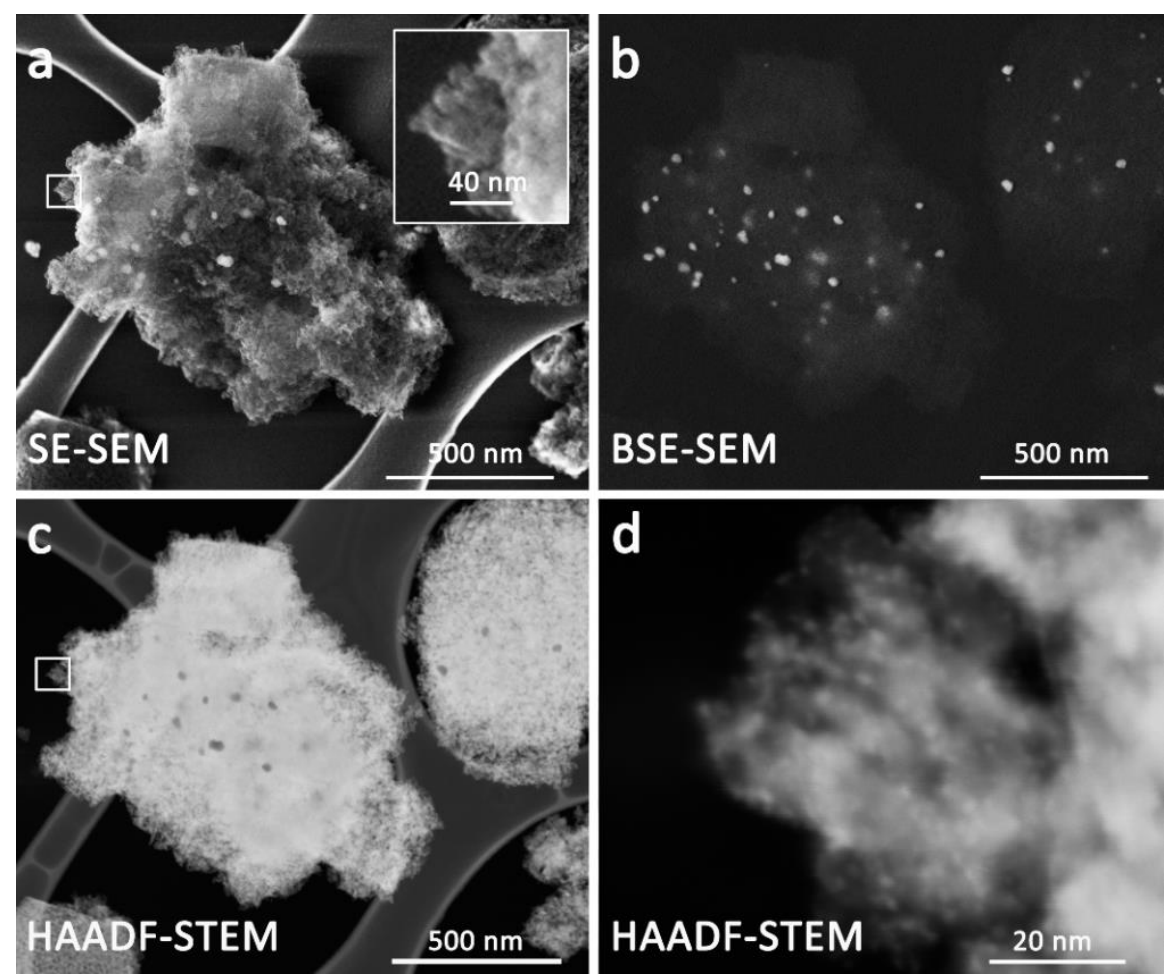

Figure 1. Pt nanoparticles on a porous $\mathrm{Al}_{2} \mathrm{O}_{3}$ carrier imaged at $30 \mathrm{keV}$ by a) SE-SEM, b) in-lens BSE-SEM, c,d) overview and high-magnification HAADF-STEM.

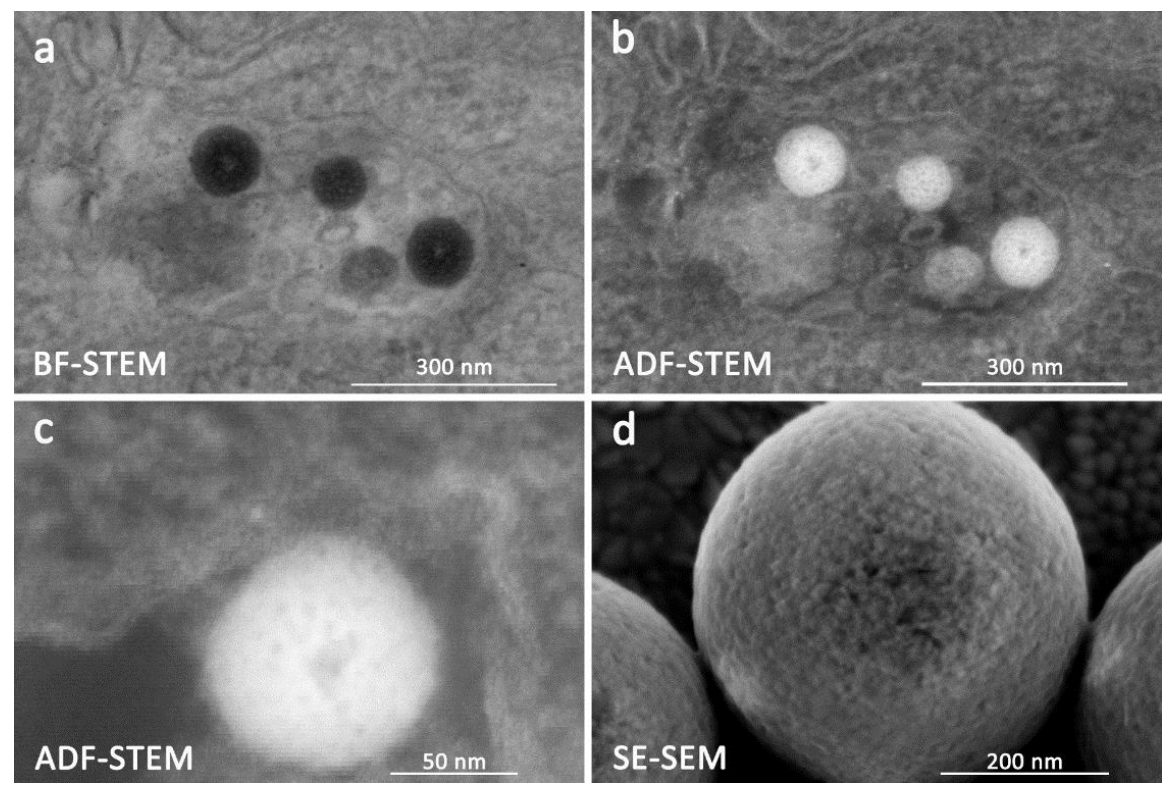

Figure 2. Thin section of an A549 cell incubated with $\mathrm{SiO}_{2}$ particles imaged by a) BF-STEM and b) ADF-STEM, c) High-magnification ADF-STEM image of an incubated $\mathrm{SiO}_{2}$ particle, d) SE-SEM topography image of another $\mathrm{SiO}_{2}$ particle. 